\title{
REFORÇO EM COMPÓSITO DE CIMENTO PORTLAND ATRAVÉS DA ADIÇÃO DE NANOTUBOS DE CARBONO DE PAREDES MÚLTIPLAS
}

\author{
Rogerio Daltro Knuth ${ }^{1}$, Margarete Regina Freitas Gonçalves ${ }^{l}$, Ricardo Marques e Silva ${ }^{1}$, Flávio Airton Knuth ${ }^{2}$ \\ ${ }^{1}$ Programa de Pós-Graduação em Ciência e Engenharia de Materiais, Universidade Federal de Pelotas, \\ 96010-000, Brasil. \\ ${ }^{2}$ Centro das Engenharias, Engenharia Civil, Universidade Federal de Pelotas, 96010-000, Brasil.
}

*E-mail: rdknuth@terra.com.br

\section{RESUMO}

A pesquisa desenvolvida no presente trabalho está embasada na aplicação da nanotecnologia a novos compósitos cimentícios, visando à melhoria das propriedades das argamassas. O objetivo do trabalho foi o de analisar o desempenho físico e mecânico de uma argamassa de revestimento produzida com nanotubos de carbono de paredes múltiplas (NTCPM), adicionados na quantidade de 0,30\% em relação à massa de cimento do compósito, comparativamente ao desempenho com uma argamassa industrializada. Para o presente estudo foi necessário funcionalizar e dispersar os NTCPM, utilizando-se os ácidos sulfúrico $\left(\mathrm{H}_{2} \mathrm{SO}_{4}\right)$ e nítrico $\left(\mathrm{HNO}_{3}\right)$, e a dispersão foi por ultrassom. Os NTCPM funcionalizados foram neutralizados com hidróxido de amônio $\left(\mathrm{NH}_{3}\right)$. Foram realizados ensaios de caracterização física e mecânica no estado fresco e no estado endurecido nas argamassas de revestimento com NTCPM e industrializada, e análise da microestrutura. Os resultados obtidos indicam que a argamassa com NTCPM apresenta melhor desempenho que a argamassa industrializada, no tocante à densidade, consistência, resistência à tração e resistência à compressão axial.

Palavras-chave: Argamassas de revestimento. Nanotubos de carbono. Compósitos. Propriedades físicas e mecânicas. Microestrutura.

\section{Introdução}

A argamassa é um dos produtos de maior utilização na construção civil, estando presente no revestimento e assentamento de diversos tipos de edificações. Quando utilizadas como revestimentos, as argamassas são fundamentais para a durabilidade dos edifícios, pois desempenham as funções de revestir e de proteger de maneira uniforme as alvenarias e estruturas de concreto contra agentes de intemperismo.

Atualmente, no mercado da construção civil predomina o emprego das argamassas de revestimento industrializadas, que apresentam maior trabalhabilidade e desempenho, por serem constituídas de materiais com controle de características tais como tamanho e quantidade de partículas.

$\mathrm{Na}$ busca pela melhor performance das argamassas, materiais como os nanotubos de carbono (NTC) vêm sendo pesquisados, visto que estes proporcionam o desenvolvimento de novos produtos com propriedades superiores aos originais, a partir de novas estruturas ou do aperfeiçoamento das já existentes.

Os NTC são estruturas formadas por átomos de carbono em arranjo hexagonal, na forma de uma ou mais folhas de grafeno enroladas de maneira concêntrica. Quando formados por uma camada são denominados nanotubos de paredes simples (NTCPS) e quando formados por duas ou mais camadas são denominados nanotubos de paredes múltiplas (NTCPM).

Os NTCPM, em função de sua baixa densidade e boa flexibilidade, são importantes componentes na obtenção de novos compósitos de argamassa. Estes apresentam o comportamento semelhante ao das fibras, que interligam os grãos constituintes das argamassas, contribuindo para a segregação e diminuição das fissuras no compósito [1].

Segundo Li et al. [2], o emprego de NTC em argamassas melhora as propriedades do compósito. Os autores, em sua pesquisa, obtiveram uma argamassa de cimento e areia com incremento de $19 \%$ na resistência à compressão e $25 \%$ na resistência à flexão com a incorporação de 0,5\% de NTCPM em relação à massa de cimento.

Focada no aprofundamento sobre o efeito da adição de NTC em compósitos de cimento, a presente pesquisa analisou o comportamento de uma argamassa de cimento, areia, plastificante e NTCPM. O desenvolvimento do trabalho foi 


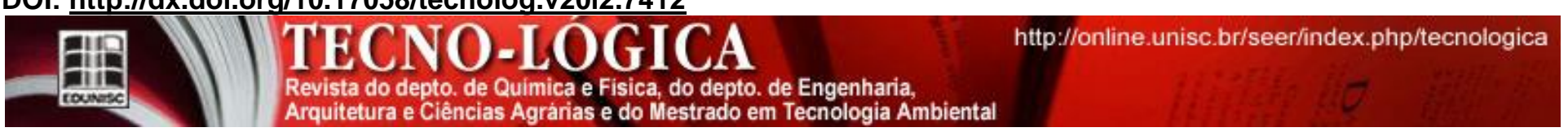

estruturado para obter o entendimento das propriedades físicas e mecânicas de uma argamassa de revestimentos aditivada com nanotubos de carbono de paredes múltiplas (NTCPM). Os resultados foram comparados com as propriedades físicas e mecânicas de argamassa industrial testada nas mesmas condições da argamassa laboratorial.

\section{Materiais e Métodos}

Para o desenvolvimento do trabalho, utilizou-se areia graduada, cimento CPV ARI, NTCPM e aditivo plastificante. A areia utilizada foi obtida comercialmente na região de Pelotas, seca em estufa na temperatura de $110^{\circ} \mathrm{C}$, por 48 horas, e preparada de acordo com a NBR NM 248 [3]. Para o trabalho utilizou-se a areia média passante na peneira \# $\mathrm{n}^{\circ} 30$ $(0,6 \mathrm{~mm})$, conforme prevê a NBR 7215 [4]. O cimento Portland utilizado foi o cimento Cimpor CPV ARI-RS e o aditivo plastificante utilizado foi o Alvenarit, ambos adquiridos comercialmente.

Os NTCPM utilizados também foram adquiridos comercialmente. Para facilitar a dispersão e melhorar a performance na massa, os NTCPM foram preliminarmente funcionalizados em solução aquosa com $30 \mathrm{~mL}$ de ácido sulfúrico $\left(\mathrm{H}_{2} \mathrm{SO}_{4}\right)$ e $10 \mathrm{~mL}$ de ácido nítrico $\left(\mathrm{HNO}_{3}\right)$, e posteriormente colocados em um aparelho sonicador de banho, da marca MaxiClean 800 Unique, por um período de 45 minutos. Finalmente, fez-se a neutralização com hidróxido de amônio $\left(\mathrm{NH}_{3}\right)$ a partir de lavagens sucessivas com água destilada até atingir o pH no intervalo de 7 a 8 . A análise da estrutura molecular dos NTCPM antes e após o processo de funcionalização foi determinada por espectroscopia Raman, na qual o espectrômetro utilizado foi um Acton Spectral pro 500 a $632,8 \mathrm{~nm}$ laser $(\mathrm{He}-\mathrm{Ne})$, e as análises térmicas foram realizadas em um analisador ATG/SDTA 851e Mettler Toledo. As amostras foram avaliadas na faixa de temperatura entre 70 e $850^{\circ} \mathrm{C}$ com uma taxa de aquecimento de $5 \mathrm{~K} / \mathrm{min}$, sob um fluxo de ar contínuo.

\subsection{Composição das argamassas}

Para o desenvolvimento do trabalho, foram preparadas a argamassa com nanotubos de carbono, identificada como ANTCPM, e a argamassa industrializada, identificada como AI. As Tabelas 1 e 2 apresentam as quantidades de materiais utilizados. O fator água/cimento para a argamassa com NTCPM foi de 1,3. Para a argamassa AI, utilizou-se a quantidade de água indicada pelo fabricante, calculada com fator água/argamassa, no valor de 0,14.
Tabela 1 - Composição da argamassa com nanotubos (ANTCPM).

\begin{tabular}{l|c|c|c}
\hline Material & Quantitativo & Unidade & $\%$ Peso \\
\hline Areia média & $2.445,50$ & $\mathrm{~g}$ & 75,99 \\
Cimento & 335 & $\mathrm{~g}$ & 10,41 \\
TCPM (funcionalizado) & 1,0 & $\mathrm{~g}$ & 0,30 \\
Aditivo & 1,35 & $\mathrm{~mL}$ & 0,04 \\
Água & 435,5 & $\mathrm{~mL}$ & 13,53 \\
\hline
\end{tabular}

Tabela 2 - Composição da argamassa industrializada (AI).

\begin{tabular}{l|c|c|c}
\hline Tabela 2 - Composição da argamassa industrializada (AI). \\
Material & Quantitativo & Unidade & $\%$ Peso \\
\hline Argamassa industrializada & $2.500,00$ & $\mathrm{~g}$ & 87,35 \\
Água & 362,00 & $\mathrm{~mL}$ & 12,65 \\
\hline
\end{tabular}

A quantidade de aditivo plastificante utilizada na argamassa ANTCPM seguiu as especificações do fabricante e a quantidade de NTCPM foi definida de acordo com o proposto por Melo [1], que demonstrou em sua pesquisa que a quantidade de $0,30 \%$ de NTC, em massa de cimento, é a que apresenta melhor desempenho em seus compósitos estudados.

As proporções em volume do aditivo e da água foram convertidas em massa com as quantidades de material seco fixas, tendo como referência o índice de consistência aproximado de $250 \pm 10 \mathrm{~mm}$, atendendo, dessa forma, a recomendação da norma NBR 13276 [5], para dosagem da proporção de água/materiais secos. Em função disto, utilizouse o fator água/cimento igual a 1,3 para a confecção das argamassas.

\subsection{Caracterização das argamassas}

Para a análise das argamassas ANTCPM e AI, foram confeccionados corpos de prova cilíndricos (dimensões de 50 mm x $100 \mathrm{~mm}$, diâmetro e altura, respectivamente), conforme a NBR 7215, e corpos de prova prismáticos (dimensões de 40 $\mathrm{mm} \times 40 \mathrm{~mm} \times 160 \mathrm{~mm}$, altura, largura e comprimento, respectivamente), conforme a NBR 13279 [6]. A caracterização foi realizada por meio do ensaio de corpos de prova com tempo de cura de 28 dias.

Nas argamassas analisaram-se as propriedades físicas, mecânicas e a sua estrutura microscópica. Para tanto, na argamassa no estado fresco determinou-se a densidade e o teor de ar incorporado conforme a NBR 13278 [7] e o índice de consistência de acordo com a NBR 13276 [5]. Na argamassa no estado endurecido determinou-se a densidade aparente de acordo com a NBR 13280 [8], a absorção de água com a NBR 9778 [9], a resistência à tração na flexão com a NBR 13279 e a resistência à compressão de acordo com a NBR 7215 [4]. Na análise dos resultados determinou-se o desvio padrão das amostras.

Também se analisou a microestrutura da argamassa com NTCPM em microscópio eletrônico de varredura da marca Jeol modelo JSM - 6610LV, existente no Centro de 
Microscopia Eletrônica do Sul (CEME-SUL), localizado no Campus Carreiros da Universidade Federal do Rio Grande (FURG).

\section{Resultados e Discussões}

\subsection{Caracterização dos NTCPM}

A Tabela 3 e a Figura 1 apresentam a caracterização de seis amostras de NTCPM funcionalizados e não funcionalizados.

A Figura 1 mostra a sobreposição da média das amostras que apresentam bandas de absorção em mesma frequência, porém com variações em suas intensidades. Segundo Belin et al.[10], podem-se resumir as características destes materiais de acordo com seu espectro, conforme mostrado na Figura 1, onde uma banda característica com o grau de desordem, denominada $D$, é caracterizada por um pico com máximo em torno de $1.340 \mathrm{~cm}^{-1}$ e a banda de alta frequência entre 1.500 e $1.600 \mathrm{~cm}^{-1}$, chamada banda $G$, é associada à qualidade dos nanotubos. Através da spectroscopia Raman, a qualidade do material foi avaliada, utilizando a razão entre as intensidades das bandas $D$ e $G\left(\mathrm{I}_{\mathrm{D}} / \mathrm{I}_{\mathrm{G}}\right)$ que representa a proporção de nanotubos perfeitos numa amostra. Quanto menor for esta razão, maior é o grau de cristalinidade do material e, portanto, melhor é a qualidade dos nanotubos.

Tabela 3 - Razão $\mathrm{I}_{\mathrm{D}} / \mathrm{I}_{\mathrm{G}}$ média de NTCPM funcionalizados e não funcionalizados.

\begin{tabular}{l|c}
\hline Nanotubos & $\mathrm{I}_{\mathrm{D}} / \mathrm{I}_{\mathrm{G} \text { média }}$ \\
\hline NTCPM $_{\text {não funcionalizado }}$ & 1,18 \\
NTCPM $_{\text {funcionalizado (H2SO4_HNO3) }}$ & 1,32 \\
\hline
\end{tabular}

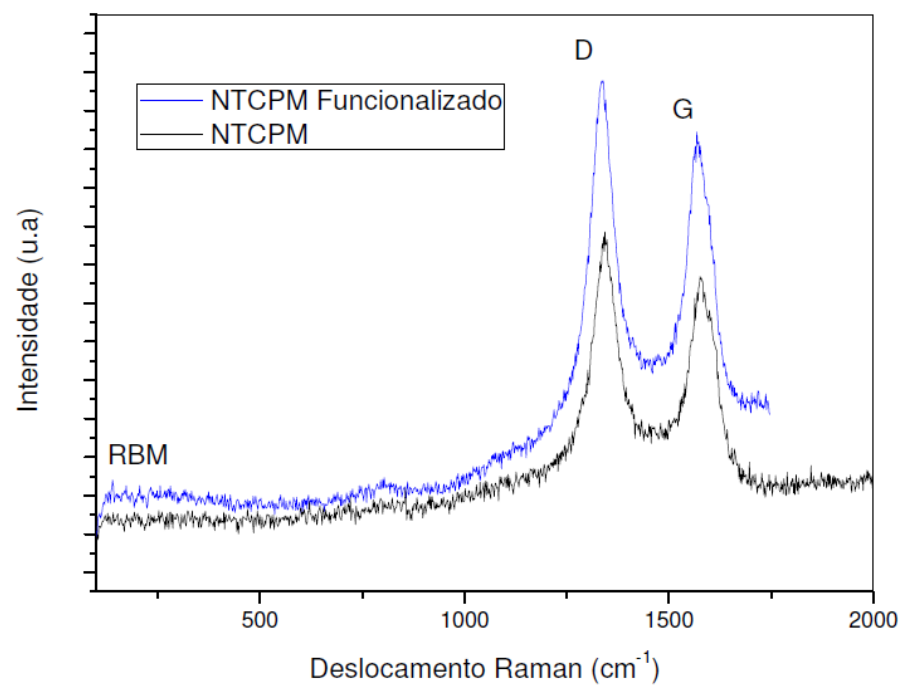

Figura 1 - Espectro Raman de NTCPM funcionalizados com ácidos sulfúrico e nítrico, comparados com NTCPM não funcionalizados.
Para a análise de possíveis defeitos estruturais dos NTC, deve-se observar se a relação entre as intensidades das bandas $D$ e $G\left(\mathrm{I}_{\mathrm{D}} / \mathrm{I}_{\mathrm{G}}\right)$ foi modificada durante o tratamento químico, provocando oxidação, quebra ou rompimento das paredes e extremidades (refletido no aumento da intensidade da banda $D$ e redução da intensidade da banda $G$ ).

Em suas pesquisas, Stobinski et al. [11], mostraram que nanotubos de carbono funcionalizados com ácido nítrico apresentaram uma redução na intensidade da banda $G$, quando comparada à banda $D$. Estas alterações nas bandas podem ser atribuídas ao aumento do número de átomos de carbono com hibridização $\mathrm{sp}^{3}$ no material. Mas a redução da intensidade da banda $G$ indica alterações na estrutura eletrônica do NTCPM como consequência, por exemplo, de diferentes quantidades de grupos funcionais presentes nas paredes e extremidades dos tubos.

Observando-se os valores da razão $\mathrm{I}_{\mathrm{D}} / \mathrm{I}_{\mathrm{G}}$ média dos NTCPM utilizados e os espectros obtidos, verifica-se que há redução na intensidade da banda $G$ em relação à banda $D$, indicando que o processo de funcionalização em meio ácido não compromete a performance dos NTCPM para as propriedades mecânicas do compósito proposto.

\subsection{Caracterização das argamassas ANTCPM e AI}

A Figura 2 apresenta corpos de prova obtidos com as argamassas ANTCPM e AI. Como se pode ver, a presença dos nanotubos de carbono interfere na tonalidade da argamassa, tornando-a mais escura.

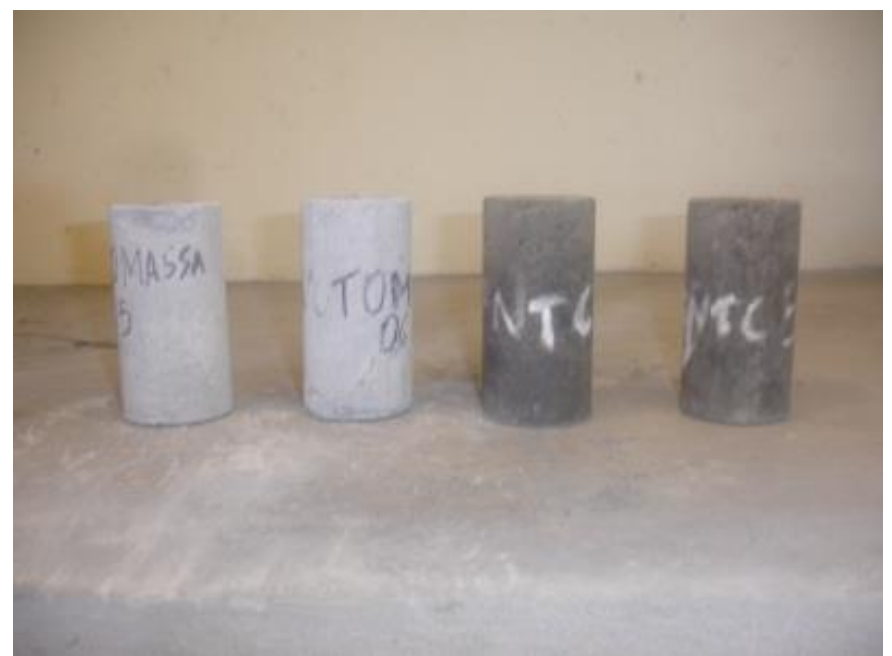

Figura 2 - Corpos de prova confeccionados com argamassa AI (cinza claro) e com argamassa ANTCPM (cinza escuro), com 28 dias de idade.

A Tabela 4 apresenta, comparativamente, os resultados de caracterização das argamassas ANTCPM e AI no estado fresco. Os resultados representam a média de seis corpos de prova. 
Tabela 4 - Caracterização das argamassas de revestimento no estado fresco.

\begin{tabular}{c|c|c|c}
\hline Argamassas & $\begin{array}{c}\text { Densidade } \\
\left(\mathrm{g} / \mathrm{cm}^{3}\right)\end{array}$ & $\begin{array}{c}\text { Teor de ar } \\
\text { incorporado }(\%)\end{array}$ & $\begin{array}{c}\text { Índice de } \\
\text { consistência }(\mathrm{mm})\end{array}$ \\
\hline ANTCPM & 1,78 & 7,0 & $240\left(\mathrm{DP}^{*}=2\right)$ \\
AI & 1,68 & 15,0 & $225\left(\mathrm{DP}^{*}=2\right)$ \\
\hline
\end{tabular}

* Desvio padrão

Os resultados obtidos mostram que, comparando com a argamassa AI, a adição de NTCPM na argamassa testada propiciou um incremento de $6 \%$ na densidade, $6,6 \%$ no índice de consistência e uma redução de $53 \%$ do percentual de ar incorporado. A justificativa para tal fato está, provavelmente, baseada nas diferenças em parâmetros relacionados ao teor de ar incorporado que interferem diretamente na densidade da massa. Isso evidencia a influência dos aditivos incorporadores de ar nas argamassas industrializadas. Segundo Bauer et al. [12], a incorporação de ar confere à estruturação interna da argamassa industrializada condição suficiente para que ela absorva os impactos sofridos durante o ensaio da mesa de consistência. Esta estruturação não é visível na argamassa confeccionada com NTCPM, uma vez que prevalece o contato íntimo entre as partículas, o que acaba por favorecer o espalhamento deste compósito durante o ensaio.

As Tabelas 5 e 6 apresentam, comparativamente, os resultados de caracterização física e mecânica das argamassas ANTCPM e AI no estado endurecido. Os resultados representam a média de seis corpos de prova com 28 dias de cura.

Tabela 5 - Propriedades físicas das argamassas de revestimento.

\begin{tabular}{c|c|c}
\hline Argamassas & Densidade $\left(\mathrm{g} / \mathrm{cm}^{3}\right)$ & Absorção de água $(\%)$ \\
\hline ANTCPM & $1,73\left(\mathrm{DP}^{*}=0,02\right)$ & $15,55\left(\mathrm{DP}^{*}=0,22\right)$ \\
AI & $1,61\left(\mathrm{DP}^{*}=0,01\right)$ & $13,35\left(\mathrm{DP}^{*}=0,20\right)$ \\
\hline
\end{tabular}

* Desvio padrão

Como se pode ver na Tabela 5, as argamassas testadas não apresentaram variação significativa da densidade. Além disso, contrariando os estudos de Marcondes [13], o qual constatou que na adição de NTC em concretos de cimento Portland há redução da absorção de água por imersão e capilaridade, a argamassa com NTCPM foi a que apresentou maior absorção de água. A justificativa para este resultado pode estar relacionada à possibilidade de falhas na dispersão dos NTCPM que, quando ocorre, permite a sua aglomeração, o que pode gerar poros de maior tamanho.

Tabela 6 - Propriedades mecânicas das argamassas de revestimento.

\begin{tabular}{c|c|c}
\hline Argamassas & Tração na flexão $(\mathrm{MPa})$ & Compressão axial $(\mathrm{MPa})$ \\
\hline ANTCPM & $3,78\left(\mathrm{DP}^{*}=0,26\right)$ & $15,55\left(\mathrm{DP}^{*}=0,12\right)$ \\
AI & $3,39\left(\mathrm{DP}^{*}=0,39\right)$ & $13,35\left(\mathrm{DP}^{*}=0,11\right)$ \\
\hline
\end{tabular}

* Desvio padrão

Os resultados da Tabela 6 mostram que a resistência à tração na flexão da argamassa com NTCPM foi $12 \%$ maior que a obtida na argamassa industrializada. Todavia, esta diferença é pequena frente aos resultados encontrados em outros estudos com NTCPM que atingiram cerca de $25 \%$ de ganhos [2]. Tal redução de ganhos pode estar ligada à diferença entre o comprimento e o diâmetro dos NTCPM utilizados que, conforme ilustrado na Tabela 7 , possui um fator de forma (relação entre comprimento/diâmetro) diferente do proposto por outros pesquisadores [2,13,14,15]. Este resultado vem reforçar a hipótese de que esse fator é relevante na resistência à tração na flexão, pois, quanto maior for o comprimento dos nanotubos, maiores serão a interação e a ancoragem com a matriz de cimento, formando, ao longo da massa cimentícia, um fenômeno, cujo comportamento se assemelha a uma microarmadura inserida no compósito, aumentando, portanto, a tenacidade na fratura [1].

Tabela 7 - Fator de forma (1/d) de nanotubos de carbono.

\begin{tabular}{l|c|c|c|c}
\hline Parâmetro & $\begin{array}{c}\text { Usados na } \\
\text { pesquisa }\end{array}$ & $\begin{array}{c}\text { Melo } \\
(2009)\end{array}$ & $\begin{array}{c}\text { Li et al. } \\
(2005)\end{array}$ & $\begin{array}{c}\text { Marcondes } \\
(2012)\end{array}$ \\
\hline $\mathrm{d}(\mathrm{nm})$ & $5,5-6,6^{*}$ & $10-100$ & $10-30$ & 9,5 \\
$\mathrm{l}(\mu \mathrm{m})$ & $5^{*}$ & $800-900$ & $0,5-500$ & 1,5 \\
Fator forma & $758-909$ & $8.000-90.000$ & $17-50.000$ & 158 \\
\hline
\end{tabular}

*Fonte: Fabricante, 2014.

Nos resultados da Tabela 6, observa-se que a argamassa com NTCPM obteve acréscimo em torno de $15 \%$ no valor de sua resistência à compressão axial em relação à argamassa industrializada. Assim como para a resistência à tração na flexão, o percentual de ganho obtido na argamassa com NTCPM é inferior ao alcançado por outros pesquisadores, como por exemplo, Li et al. [2], que, em seus experimentos, obtiveram um acréscimo de até $19 \%$ na resistência à compressão. Este resultado se justifica pela maior absorção de água do compósito que, como anteriormente falado, possivelmente tenha sido causada pela presença de poros de maior tamanho, formados pela inadequada distribuição dos NTCPM. Esta hipótese pode ser comprovada a partir da micrografia apresentada na Figura 3, onde é possível visualizar os NTCPM aglomerados no entorno de um poro.

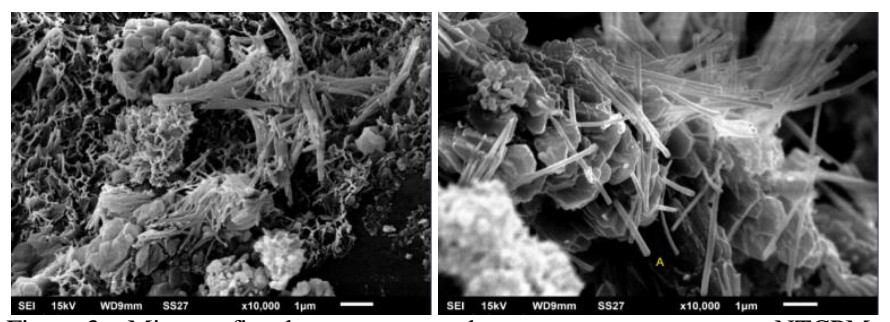

Figura 3 - Micrografias de uma amostra da argamassa composta por NTCPM.

\section{Conclusões}

Por meio dos resultados obtidos e analisados, pode-se afirmar que:

A inserção de NTCPM em argamassa de cimento melhora o desempenho mecânico do compósito. 
$\mathrm{O}$ percentual de $0,30 \%$ de NTCPM adicionado à argamassa resultou em ganhos de resistência à tração na flexão e à compressão, em relação à argamassa industrial.

O melhor desempenho da argamassa com adição de NTCPM pode ser atribuído às redes, malhas e densificação formadas pelos NTCPM, que possibilitaram a redução na propagação de fissuras.

\section{INCREASE IN PORTLAND CEMENT COMPOSITE THROUGH ADDITION OFF CNTs OFF MULTIPLE WALLS}

\begin{abstract}
The research developed in this work is grounded in the application of nanotechnology to new cementitious composites in order to improve the properties of mortars. The objective was to analyze the physical and mechanical performance of a coating mortar produced with multi-walled carbon nanotubes (NTCPM), added in the amount of $0.30 \%$ in relation to the concrete mass of the composite compared to performance with industrialized mortar. For the present study it was necessary to functionalize and disperse NTCPM, using sulfuric acid (H2SO4) and nitric (HNO3), and it was dispersed by ultrasound. The functionalized NTCPM were neutralized with ammonium hydroxide (NH3). Physical and mechanical characterization tests were performed in fresh and hardened state in coating mortars NTCPM and industrialized ones and it was analyzed the microstructures. The results indicate that the mortar with NTCPM outperforms the industrialized mortar, with respect to density, consistency, tensile strength and resistance to axial compression.
\end{abstract}

Keywords: Coating mortars. CNTs. Composites. Physical and mechanical properties. Microstructure.

\section{Referências}

[1] MELO, V. S. Nanotecnologia aplicada ao concreto: efeito da mistura física de nanotubos de carbono em matrizes de cimento Portland. 2009. 146f. Dissertação (Mestrado em Engenharia, área de concentração materiais de Construção civil). Universidade Federal de Minas Gerais, Belo Horizonte, 2009.

[2] LI, G. Y.; WANG, P. M.; ZHAO, X. Mechanical behavior and microstructure of cement composites incorporating surface-treated multi- walledcarbon nanotubos. Carbon, n. 43, p. 1239-45, 2005. Disponível em: <http://www.sciencedirect.com>. Acesso em: 20 mar. 2014.

[3] ABNT - Associação Brasileira de Normas Técnicas. NBR NM 248. Agregados - Determinação da composição granulométrica. 2003. 6p.

[4] ABNT - Associação Brasileira de Normas Técnicas. NBR 7215. Cimento Portland - Determinação da resistência à compressão. Rio de Janeiro, 1997. $8 \mathrm{p}$.

[5] ABNT - Associação Brasileira de Normas Técnicas. NBR 13276. Argamassa para assentamento e revestimento de paredes e tetos - Preparo da mistura e determinação do índice de consistência. Rio de Janeiro, 2005. 3p.

[6] ABNT - Associação Brasileira de Normas Técnicas. NBR 13279. Argamassa para assentamento e revestimento de paredes e tetos Determinação da resistência à tração na flexão e à compressão. Rio de Janeiro, 2005. 9p.

[7] ABNT - Associação Brasileira de Normas Técnicas. NBR 13278. Argamassa para assentamento e revestimento de paredes e tetos Determinação da densidade de massa e do teor de ar incorporado. Rio de Janeiro, 2005. 4p.

[8] ABNT - Associação Brasileira de Normas Técnicas. NBR 13280. Argamassa para assentamento e revestimento de paredes e tetos Determinação da densidade de massa aparente no estado endurecido. Rio de Janeiro, 2005. 2p.

[9] ABNT - Associação Brasileira de Normas Técnicas. NBR 9778. Argamassa para assentamento e revestimento de paredes e tetos Determinação da densidade de massa e do teor de ar incorporado. Rio de Janeiro, 2005. 4p.

[10] BELIN, T., EPRN, F., 2005, "Characterization Methods of Carbon Nanotubes: A Review", Materials Science and Engineering B, v. 119, pp. 105118.

[11] STOBINSKI, L.; LESIAK, B.; KOVER, L.; TOTH, J.; BINIAK, S.; TRYKOWSKI, G.; JUDEK, J. Multiwall carbon nanotubes purification and oxidation by nitric acid studied by the FTIR and electron spectroscopy methods, Journal of Alloys and Compounds, v. 501, n1, p. 77-84. 2010.

[12] BAUER, E. ; SOUSA, J. G. G. Materiais constituintes e suas funções. In: BAUER, E. (Org.). Revestimentos em Argamassas: características e peculiaridades. Brasília, LEM-UnB; SINDUSCON/DF, 2005. p. 15-22.

[13] MARCONDES, C. G. N. Adição de nanotubos de carbono em concretos de cimento Portland - absorção, permeabilidade, penetração de cloretos e propriedades mecânicas. 2012. 143f. Dissertação (Mestrado em Engenharia de Construção Civil). Universidade Federal do Paraná. Curitiba, 2012.

[14] BATISTON, E. R. Estudo exploratório dos efeitos de nanotubos de carbono em matriz de cimento. 2007. 80f. Dissertação (Mestrado em Engenharia Civil). Universidade Federal de Santa Catarina, Florianópolis, 2007.

[15] YAKOVLEV, G.; KERIENÉ, J.; GAILIUS, A.; GIRNIENÉ, I. Cement based foam concrete reinforced by carbon nanotubes. Materials Science, v.12, n2, p.147-151, 2006. Disponível em: <http://www.ktu.lt/lt/mokslas/zurnalai/ medz/medz0-85/12ceramics $>$. Acesso em: 12 fev. 2014. 